\title{
A Case of Supraventricular Tachycardia Associated With Wolff-Parkinson-White Syndrome
}

\author{
Yong Chuan Chee ${ }^{\mathrm{a}, \mathrm{b}}$, Jian-Gang Ang ${ }^{\mathrm{a}}$
}

\begin{abstract}
Wolff-Parkinson-White syndrome (WPWS) is the most common form of ventricular pre-excitation. It is characterized by the presence of an accessory pathway between the atrium and ventricles which allows an alternative route for ventricular depolarization. Although most WPWS remain asymptomatic, others are prone to tachyarrhythmias that can be life-threatening. WPWS can present with variable tachyarrhythmias that require specific treatment considerations. We report a case of WPWS that was diagnosed following successful termination of narrow complex tachycardia. This is followed by a review of literature covering the diagnostic and initial therapeutic issues. Adenosine, a commonly used atrioventricular (AV) nodal blocking agent, is considered as the first-line therapeutic agent, which terminates regular narrow complex tachycardia. Clinicians need to be aware of the potential for adenosine to induce lethal tachyarrhythmias via AV nodal blockade and preferential conduction down the accessory pathway in patients with pre-excitation syndrome.
\end{abstract}

Keywords: Wolff-Parkinson-White; Pre-excitation; Adenosine; AV blocker; Supraventricular tachycardia

\section{Introduction}

The majority of patients having the Wolff-Parkinson-White (WPW) pattern on the electrocardiogram (ECG) remain asymptomatic. However, others are prone to tachyarrhythmias that can be life-threatening. The WPW syndrome may remain undetected until it manifests as a paroxysmal supraventricular tachycardia (PSVT) and it comprises around $2.4 \%$ of patients presenting with narrow complex tachycardia to the emergency department where it can be successfully treated with restoration of sinus rhythm [1]. We present a case where florid features of pre-excitation were detected on a 12-lead ECG follow-

Manuscript submitted October 17, 2017, accepted November 18, 2017

aDepartment of Medicine, Hospital Sultanah Bahiyah, Alor Setar, Kedah, Malaysia

${ }^{b}$ Corresponding Author: Yong Chuan Chee, Hospital Sultanah Bahiyah, KM 6, Jalan Langgar, Alor Setar, Kedah, Malaysia.

Email: cheeyongchuan@gmail.com

doi: https://doi.org/10.14740/jmc2693w ing a successful management of supraventricular tachycardia (SVT). We also cautioned on the use of atrioventricular (AV) nodal blocking agents like adenosine in patients with preexisting ECG evidence of pre-excitation and reiterated on the importance of re-examining the ECG following acute management of arrhythmia.

\section{Case Report}

A 40-year-old lady with no significant medical illnesses presented with a sudden onset of palpitation for the past hour prior to admission to the emergency department. The palpitation was associated with mild shortness of breath and reduced effort tolerance. This was her first visit to the hospital for the above complaint. She was previously well with no fever, cough, chest pain or syncopal attack. She denied smoking, taking alcohol or being involved with illicit drug use. She recalled having similar episodes 3 years ago whereby she had transient loss of consciousness. There was no family history of sudden or premature cardiovascular death. Physical examination was otherwise unremarkable with normal heart and lung sounds. She was afebrile and her vital signs were normal. The cardiac monitor revealed narrow complex tachycardia at approximately 160 beats per minute; a 12-lead ECG was performed.

\section{Interpretation}

Upon arrival, her ECG showed a regular narrow complex tachycardia with a heart rate of 160 beats per minute. The axis was normal with no acute ST segment changes. The P waves can best be seen embedded in the ST segment of lead III and aVF with a short RP interval. Non-specific ST segment depression and $\mathrm{T}$ wave inversion could be seen over the infero-lateral leads (Fig. 1).

\section{Clinical course}

The patient remained alert, awake with normal blood pressure. A trial of carotid sinus massage and Valsalva maneuver did not revert the rhythm nor slow down her ventricular rate. Therefore, IV adenosine $(6 \mathrm{mg})$ was subsequently delivered in an effort to covert the rhythm. She was stable throughout the procedure with no signs of poor perfusion. She was successfully cardioverted and a repeated ECG following cardiover- 


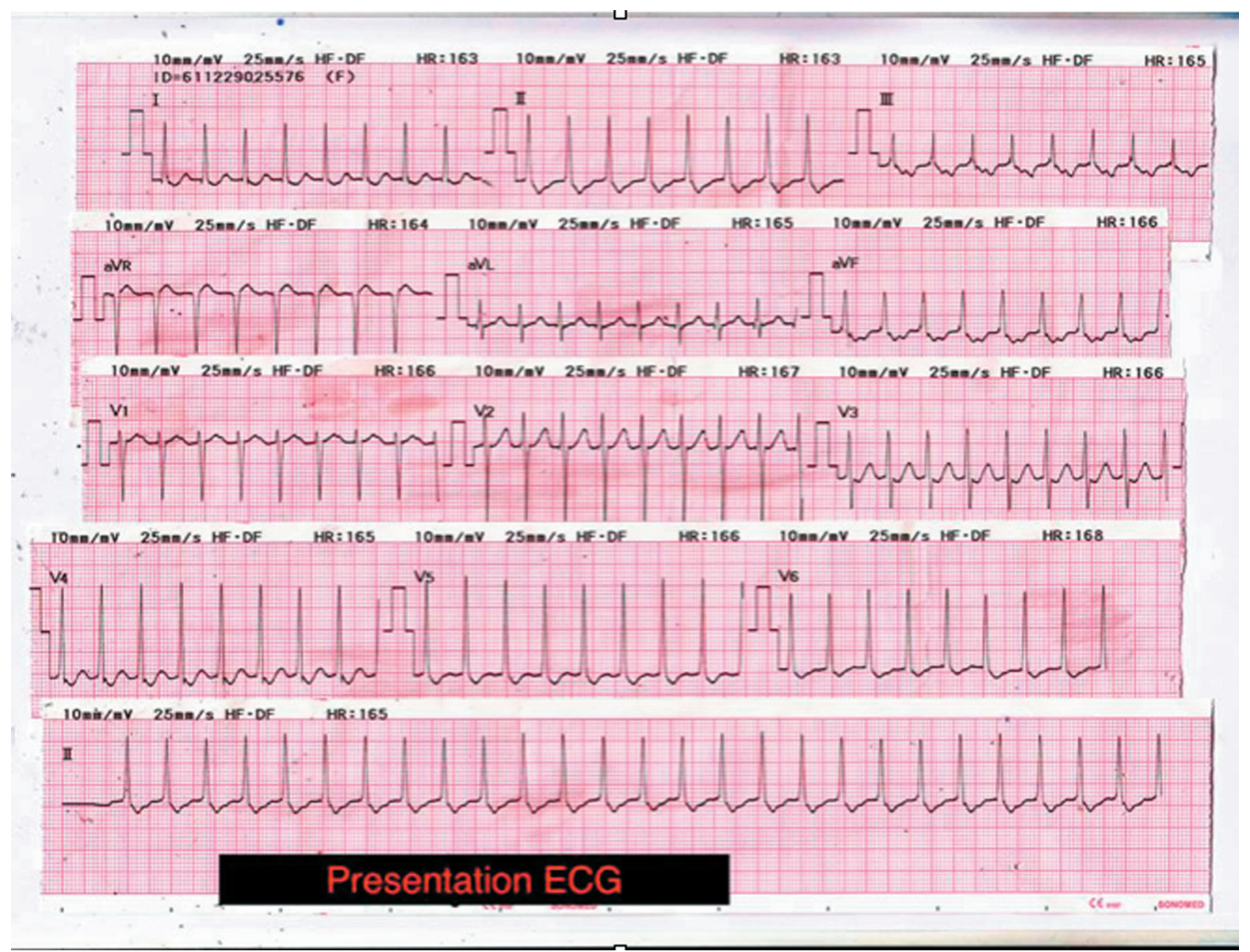

Figure 1. ECG upon arrival shows regular narrow complex tachycardia beating at a rate of 160 beats/min. Axis is normal with no acute ST segment changes. Non-specific ST segment depression and T wave inversion can be seen over the infero-lateral leads. Inverted P waves may be visible de-forming the ST segment indicating that atrial depolarisation occurs later than ventricular depolarisa-tion. In a patient with a narrow complex tachycardia the presence of such late $\mathrm{P}$ waves is frequently the only ECG evidence that the patient has an accessory pathway rather than a much more common atrioventricular nodal re-entrant tachycardia (AVNRT).

sion showed florid features of pre-excitation.

ECG findings post chemical cardioversion revealed a sinus rhythm as evidenced by the presence of upright $P$ waves in lead II preceding the QRS complexes. There was a shortened PR interval with distinct slurred upstroke of the ascending limb of the QRS complexes, which was best appreciated over the limb leads. The QRS complexes were slightly widened. There were also repolarization changes over the limb and precordial leads in the form of discordant ST segment deviation. In addition, the presence of Q waves over the inferior leads gave rise to a pseudo-infarction pattern (Fig. 2). The overall features were in keeping with the presence of pre-excitation syndrome (WPW syndrome). The patient was subsequently referred to an electrophysiologist for radiofrequency ablation to prevent recurrent dysrhythmias or a sudden cardiac death.

\section{Discussion}

In 1930, Louis Wolff, Sir John Parkinson, and Paul Dudley White first reported 11 patients who suffered from recurrent attacks of tachycardia, which was associated with an ECG pat- tern of bundle branch block with short PR interval. This was subsequently termed as WPW syndrome [2].

Atrioventricular re-entrant tachycardia (AVRT) is a reentrant tachycardia that occurs in WPW syndrome. In AVRT, the normal AV node and an accessory pathway along with the atria and ventricles form a circuit that allows impulse re-entry. There exist two forms of AVRT: orthodromic and antidromic. In orthodromic AVRT, the atrial impulse is conducted to the ventricles via the normal AV node route and is conducted retrogradely via an accessory pathway allowing the impulse to re-enter the conduction system and perpetuate the tachycardia.

Orthodromic AVRT comprises $90-95 \%$ of the re-entrant tachycardias which are associated with WPW and can be initiated by atrial or ventricular premature beats $[2,3]$. The ECG during orthodromic AVRT typically shows a ventricular rate ranging from 150 to 250 beats per minute and is usually regular. The QRS complexes are usually narrow in the absence of underlying conduction system disease or aberrancy. Inverted $\mathrm{P}$ waves with an RP interval that is usually less than one-half of the RR interval can also be seen. Ischemic appearing ST segment depression may also occur during orthodromic AVRT, 


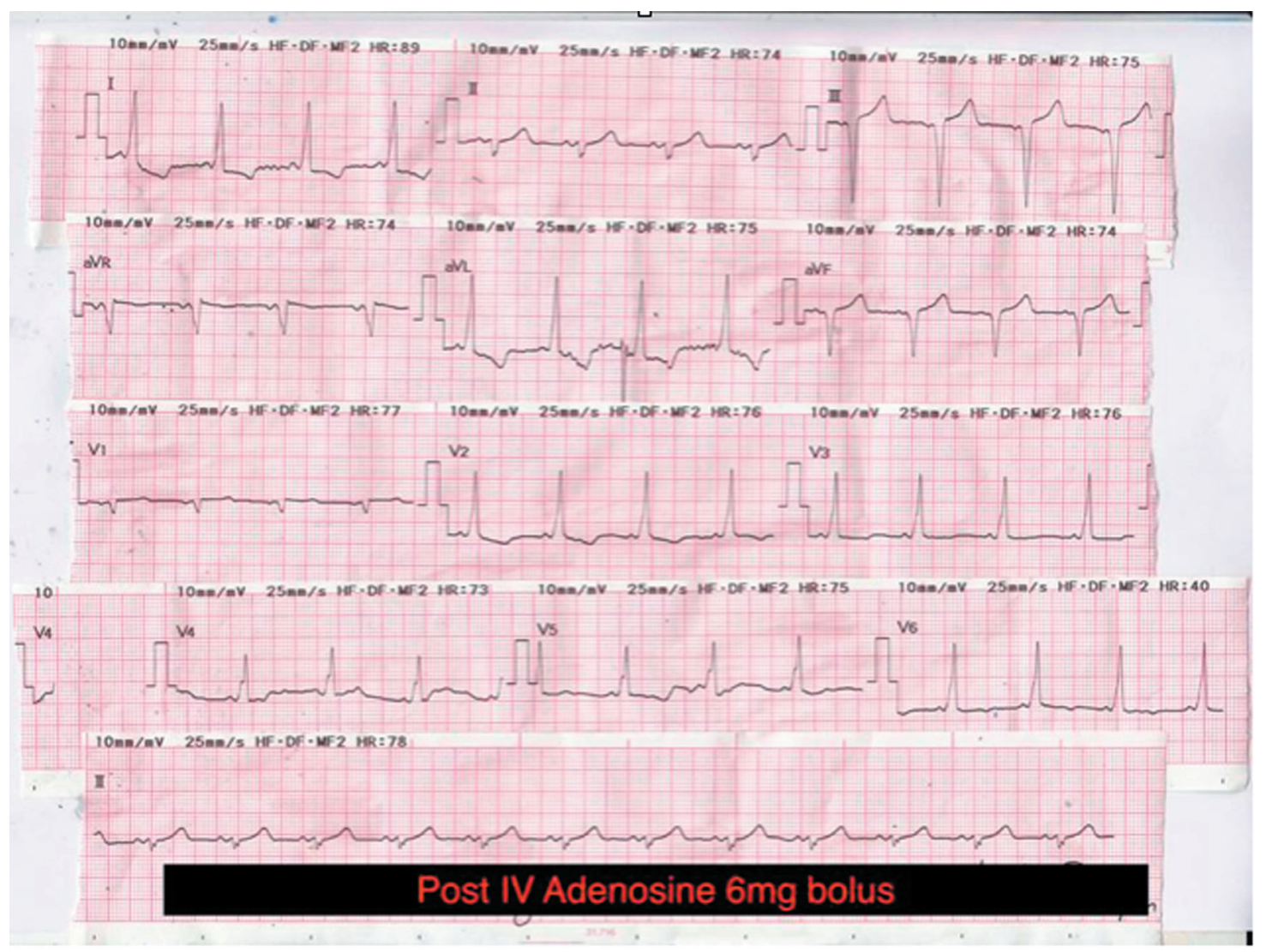

Figure 2. The classic triad of electrocardiographic findings in Wolff-Parkinson-White syndrome: short PR interval, broad QRS complex and delta wave.

even in the absence of coronary artery disease. Several factors may contribute to the ST segment depression in these arrhythmias, including changes in autonomic nervous system tone, intraventricular conduction disturbances, a longer ventriculo-atrial interval, and a retrograde $\mathrm{P}$ wave of longer duration which overlaps into the ST segment [4].

Although the classic findings for diagnosing WPW syndrome are the presence of short PR interval and delta wave with wide QRS complex, the most common dysrhythmia is narrow QRS tachycardia. Usually, it is not possible to differentiate PSVT caused by atrioventricular nodal re-entrant tachycardia (AVNRT) from PSVT caused by orthodromic AVRT because they both are rapid, narrow QRS complex tachycardia. Indicators that a PSVT may be caused by an orthodromic AVRT are ST segment depression and beat-to-beat oscillation in the QRS amplitude. An aspect that can contribute to the ECG differential diagnosis is the fact that the $\mathrm{P}$ wave in nodal reentrant tachycardia is usually within the QRS complex or at the end, whereas in accessory pathway mediated SVT, the P wave is normally behind the QRS complex since the impulse has to travel through part of the ventricle and the accessory pathway before reaching the atrium. When the normal rhythm is restored like in this case, the short PR interval and delta waves may be seen.

This case is a typical presentation of SVT, successfully managed with IV adenosine following which delta waves are detected in the ECG suggestive of pre-excitation in WPW syndrome. However, it is not necessary to distinguish PSVT due to AVNRT or AVRT since both can be successfully treated with maneuvers or drugs that can block the AV nodes such as beta blocker, digoxin and calcium channel blockers. It only remains to add that in the rare event where the patient has pre-excitation during sinus rhythm, adenosine may potentially induce a preexcited atrial fibrillation, which can be fatal if sustained given the extremely rapid ventricular rate. The higher incidence of atrial fibrillation in patients with accessory pathways when compared with those with AVNRT may be caused by both the decrease in atrial refractory period due to adenosine as well as the anatomic substrate in AVRT due to arborization of the atrial accessory pathway insertion sites [5-7].

Unstable patient with WPW syndrome related atrial fibrillation is best managed with synchronized cardioversion. Medications with significant AV nodal blocking effects should be avoided in all instances. These agents may include amiodarone, calcium channel and beta blocking agents, adenosine and digoxin. Such agents can promote enhanced conduction via the accessory pathway resulting in preferential conduction down the accessory pathway precipitating an increasingly rapid ventricular rate as well as a possible cardiovascular collapse [8]. Adenosine is widely used in an acute care setting and is generally considered to be safe and effective diagnostic and therapeutic agent. More common side effects like bronchospasm, 
chest pain, and bradycardia are familiar to clinicians except for the lethal tachyarrhythmias, particularly if there is previous evidence of pre-excitation. Since the arrhythmias may be life-threatening, administration of adenosine should only be conducted when there is rapid access to defibrillation and other anti-arrhythmic agents. When a full history has been taken, medication recorded, and careful analysis of ECGs made, adenosine remains the first agent of choice for many tachyarrhythmias, although its potential to produce cardiovascular collapse on rare occasions must be borne in mind. This case also highlights the importance of following up patients following successful management of arrhythmia/SVT.

\section{Acknowledgments}

The authors would like to thank the Director General of Health Malaysia for the permission to publish this paper. We would also like to thank Datuk Dr. Muhammad Radzi Abu Hassan, Head, Department of Internal Medicine for his relentless support in our academic pursuit.

\section{Conflict of Interest}

None.

\section{Abbreviations}

WPW: Wolff-Parkinson-White; SVT: supraventricular tachycardia; AV: atrioventricular; PSVT: paroxysmal atrioventricular tachycardia; AVRT: atrioventricular re-entrant tachycardia; AVNRT: atrioventricular nodal re-entrant tachycardia; ECG: electrocardiograph

\section{References}

1. Brady WJ, Jr., DeBehnke DJ, Wickman LL, Lindbeck G. Treatment of out-of-hospital supraventricular tachycardia: adenosine vs verapamil. Acad Emerg Med. 1996;3(6):574-585.

2. Josephson ME. Preexcitation syndromes. In: Clinical Cardiac Electrophysiology, 4th, Lippincot Williams \& Wilkins, Philadelphia 2008. p. 339.

3. Chugh A, Morady F. Atrioventricular reentry and variants. In: Cardiac electrophysi- ology from cell to bedside, 5th edition, Zipes DP, Jalife J. (Eds), Saunders/Elsevier, Philadelphia 2009. p. 605-614.

4. Nelson SD, Kou WH, Annesley T, de Buitleir M, Morady F. Significance of ST segment depression during paroxysmal supraventricular tachycardia. J Am Coll Cardiol. 1988;12(2):383-387.

5. DiMarco JP, Miles W, Akhtar M, Milstein S, Sharma AD, Platia E, McGovern B, et al. Adenosine for paroxysmal supraventricular tachycardia: dose ranging and comparison with verapamil. Assessment in placebo-controlled, multicenter trials. The Adenosine for PSVT Study Group. Ann Intern Med. 1990;113(2):104-110.

6. McIntosh-Yellin NL, Drew BJ, Scheinman MM. Safety and efficacy of central intravenous bolus administration of adenosine for termination of supraventricular tachycardia. J Am Coll Cardiol. 1993;22(3):741-745.

7. Silverman AJ, Machado C, Baga JJ, Meissner MD, Lehmann MH, Steinman RT. Adenosine-induced atrial fibrillation. Am J Emerg Med. 1996;14(3):300-301.

8. Camm AJ, Kirchhof P, Lip GY, Schotten U, Savelieva I, Ernst S, Van Gelder IC, et al. Guidelines for the management of atrial fibrillation: the Task Force for the Management of Atrial Fibrillation of the European Society of Cardiology (ESC). Europace. 2010;12(10):1360-1420. 